\title{
VALIDACIÓN DE MÁQUINA HIDRÁULICA PARA LA ELABORACIÓN DE BRIQUETAS A PARTIR DE CASCARILLA DE CAFÉ Y RESIDUOS DE MADERA.
}

\section{VALIDATION OF A HYDRAULIC MACHINE FOR MAKING BRIQUETTES, FROM COFFEE HUSKS AND WOOD WASTES.}

\author{
Karla Elisabeth Dávila ${ }^{4}$ \\ Sorayda Rugama ${ }^{5}$ \\ Erick Briones ${ }^{6}$
}

RESUMEN: En el marco del Proyecto UNI - PNUD para el desarrollo de la temática "Generación de prototipos de tecnologías vinculadas al Cambio Climático", se realizó la validación de una máquina briqueteadora hidráulica para elaborar briquetas a partir de cascarilla de café y residuos de madera, para ello se determinaron los requerimientos técnico mecánicos de la máquina, se caracterizaron las briquetas elaboradas con la máquina hidráulica y por último se calcularon los costos de producción de las briquetas. En la determinación de los requerimientos técnicos - mecánicos se obtuvo que se necesita de una presión de 2 a 4 ton para cada briqueta; el equipo produce 6 briquetas y por ello se consideró utilizar una gata de 12 ton. Así mismo se determinó el diseño, material de construcción del equipo hidráulico y su nivel de productividad para compararla con la prensa manual de hierro; donde se obtuvo que el equipo hidráulico produce 6 briquetas en $6.33 \mathrm{~min}$ en cambio la prensa manual de hierro produce 6 briquetas en $23.90 \mathrm{~min}$; y por último se planteó un manual de mantenimiento y uso de la máquina. Para la caracterización de las briquetas elaboradas con la máquina hidráulica se ejecutaron diferentes parámetros entre estos: granulometría, compactación, combustión, humedad, ceniza, poder calorífico, emisión de $\mathrm{CO}_{2}$ y calculó de la productividad. Según los datos obtenidos las mezclas resultantes fueron la EXPM1, EXPM2, EXPM4 y EXPM5, de estas las más óptimas "EXPM1 y EXPM2"; y por último los costó de producción se calcularon para estos tratamientos, donde se obtuvo que el precio de producción por unidad (6 briquetas) para la EXPM1 es de C $\$ 4.51$ y para la EXPM2 es de $C \$ 4.62$, al comparar estos precios con el precio de la mezcla testigo ( $\mathrm{C} \$ 4.50)$ se concluyó que son más baratos.

PALABRAS CLAVE: Briquetas; almidón; cascarilla de café; residuos de madera (Aserrín y colocho); briqueteadoras (Máquina Hidráulica y Prensa Manual de Hierro).

ABSTRACT: In the framework of the UNI - UNDP Project for the development of the theme "Generation of technologies prototypes linked to Climate Change", a validation of a hydraulic machine for making briquettes from coffee husks and wood wastes. For this, the technical mechanical requirements of the machine were determined, the briquettes elaborated with the

\footnotetext{
${ }^{4}$ Docente, Investigadora. UNI-RUACS, Nicaragua Karda.2008@yahoo.com - autor para la correspondencia

${ }^{5}$ UNI-RUACS, Nicaragua - rugamapicado@gmail.com

${ }^{6}$ UNI-RUACS, Nicaragua - dani.lopez22@gmail.com
} 
hydraulic machine were characterized and finally the production costs of the briquettes were calculated.

For the determination of the technical - mechanical requirements it was obtained that a pressure of 2 to 4 tons is required for each briquette; the equipment produces 6 briquettes and for that reason it was considered to use a hydraulic press of 12 tons. Likewise, the design, construction material of the hydraulic equipment and its level of productivity were determined to be compared with the manual iron press; Where it was obtained that the hydraulic equipment produces 6 briquettes in 6.33 minutes instead the manual iron press produces 6 briquettes in 23.90 minutes; And finally a manual of maintenance and use of the machine was proposed.

For the characterization of the briquettes made with the hydraulic machine, different parameters were executed: granulometry, compaction, combustion, humidity, ash, calorific value, CO2 emission and Productivity calculations. According to the data obtained the resulting mixtures were EXPM1, EXPM2, EXPM4 and EXPM5, of these the most optimal "EXPM1 and EXPM2"; And finally the cost of production was calculated for these treatments, where it was obtained that the cost of production per unit ( 6 briquettes) for the EXPM1 it is $C \$ 4.51$ and for the EXPM2 it is $\mathrm{C} \$ 4.62$, when comparing these prices with The price of the control mixture ( $C \$ 4.50)$ was concluded that they are cheaper.

KEYWORDS: briquettes; starch; coffee husks; wood waste; briquetting

\section{ABREVIATURAS}

- $\quad$ CO2 (Dióxido de carbono).

- EXPM1 (Experimentación mezcla 1): Cisco sin moler (12.69 \%), aserrín (12.69 \%), almidón en polvo $(7.46 \%)$ y agua (67.16 \%).

- EXPM2 (Experimentación mezcla 2): Cisco sin moler (10.45\%), aserrín (10.45\%), colocho (4.48\%), almidón en polvo (7.46) y agua (67.16).

- EXPM4 (Experimentación mezcla 4): Cisco molido (12.69 \%), aserrín (12.69%), almidón en polvo $(7.46 \%)$ y agua $(67.16 \%)$.

- EXPM5 (Experimentación mezcla 5): Cisco molido (10.45\%), aserrín (10.45\%), colocho (4.48\%), almidón en polvo (7.46) y agua (67.16).

- Mezcla testigo: Cisco molido, almidón cocido y agua.

\section{INTRODUCCIÓN}

En la actualidad existe un alto riesgo de contaminación en Nicaragua por los residuos provenientes de las industrias alimenticias, industrias químicas y agro-industrias, ya que debido a sus características y prácticas productivas representan los sectores de mayor riesgo en cuanto a la emisión de residuos en nuestro país. Esto se debe a que quizás no toman en cuenta las normativas y las leyes establecidas para su funcionamiento, así como también de buenas prácticas de manufactura y tecnologías para mitigar el daño que estas provocan. 


\section{Karla Elisabeth Dávila; Sorayda Rugama; Erick Briones}

Por lo antes expuesto, urge que las industrias pongan en marcha planes de gestión integral, proporcionen métodos de reúso, reciclaje y reducir, tal es el caso del aprovechamiento de residuos provenientes del sector cafetalero y maderero como la cascarilla de café y residuos de madera; de los cuales se pueden aprovechar su contenido calórico para generar energía y de esta manera aminorar el impacto generado al medio ambiente, en primer lugar por quema de árboles y en segundo lugar por la contaminación que estos residuos podrían estar generando al medio.

En este sentido se han realizado estudios que demuestran la utilidad de la cascarilla de café en briquetas, así mismo se ha utilizado el aserrín en eco fogones como medio de combustible. De esa forma se aprovecha uno de los desperdicios del procesamiento del café y uno generado por las industrias madereras.

En concordancia con lo planteado, la presente investigación estuvo centrada en la validación de un prototipo de máquina hidráulica para elaborar briquetas a partir de cascarilla de café y residuos de madera, para aprovechamiento de estos como combustible; en el que se logró diseñar la máquina y variar mezclas a través de fases experimentales; las cuales permitieron mejorar el diseño y la productividad de la máquina briqueteadora actual, así como también el diseño y proporciones de mezclas de la briqueta que contribuyan a mejorar los niveles de combustión en estas.

\section{METODOLOGÍA}

La primera etapa de la investigación consistió en la realización de los requerimientos técnicos mecánicos donde se determinó la presión para la compactación de la briqueta, tomando como base la fuerza encontrada por (Amador, Rugama, \& Morazán, 2012), esto permitió conocer el tipo de presión requerida para la compactación de la mezcla. Una vez establecidos los parámetros de diseño de la máquina, se procedió a la construcción de la misma, donde luego se sometió a trabajo con una muestra testigo a fin de determinar su nivel de funcionamiento y detectar algunas fallas mecánicas y establecer correcciones en su diseño de ser necesario.

Posteriormente se llevó a cabo la caracterización de las briquetas que inició con la modificación de las mezclas, donde se plantearon una serie de combinaciones a partir de los principales residuos a utilizar "Cascarilla de café y residuos de madera", así también en esta fase de la investigación se determinó el porcentaje a utilizar de cada residuo acorde al peso de la briqueta; y se estandarizo el tiempo de descomposición de algunas de las mezclas (Ver tabla 1). Cabe mencionar que se aplicó el mismo porcentaje para todas las combinaciones y se realizó 5 repeticiones de cada una de estas utilizando la máquina hidráulica, esto con la finalidad de determinar la mezcla idónea. Por último se evaluaron las mezclas óptimas encontradas con la mezcla testigo (Cascarilla triturada y almidón cocido), a fin de comparar la eficiencia y productividad de estas a través de pruebas físicas que permitieron obtener el \% humedad, \% de cenizas, poder calorífico, emisión de $\mathrm{CO}_{2}$, tiempo de durabilidad de combustión y resistencia a la compresión. 
Y por último se calculó los costos de producción, para lo cual fue necesario determinar los costos variables, materia prima, mano de obra directa, servicios como el agua, mantenimiento, suministros e inversión inicial.

\section{RESULTADOS Y DISCUSIÓN}

Las toneladas de la gata hidráulica puede oscilar entre 2 y 4 toneladas para generar la presión comprendida entre 2.02 y $3.63 \mathrm{MPa}$, para la producción de una briqueta. El material que presentó mayores ventajas para la construcción de la máquina fue el hierro, ya que este presenta mayor durabilidad y resistencia, debido a que durante la elaboración de las briquetas, estas se realizaran en ambiente húmedo permanente, además sería posible efectuar modificaciones de diseño. Otro de los aspectos a analizar se refirieron al diseño de la máquina, incluyendo sus partes, en este sentido se determinó que la máquina estaría constituida por las siguientes piezas: cuerpo de la máquina, gata hidráulica (12 ton), resortes, placa de distribución de presión, barras de compactación, molde o grillete, placa de fijación y refuerzo, guías horizontales y embudo, como se muestra en la figura 1.

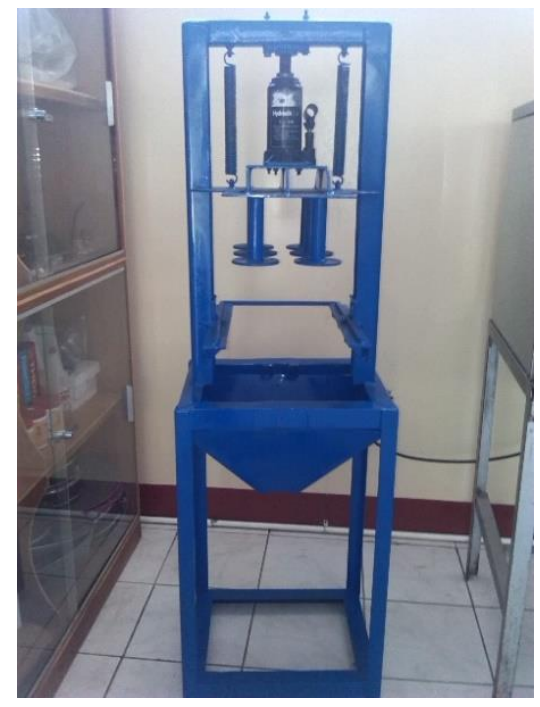

Fig. 1: Máquina Hidráulica

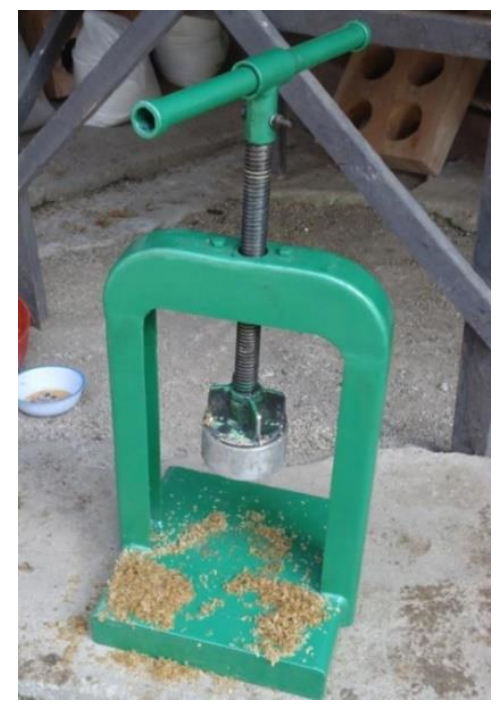

Fig. 2: Prensa Manual de Hierro

El equipo una vez construido fue sometido a pruebas de funcionamiento utilizando la mezcla testigo, donde se encontraron fallas en la extracción del producto debido a que el gato hidráulico no recorría toda la distancia y en los grilletes y en la placa de fijación y refuerzo quedaba adherida la briqueta. De acuerdo a estas fallas se plantearon modificaciones en los grilletes, en las barras de compactación y se añadió un pin de perforación que permitiera obtener la briqueta con un hueco concéntrico; todo esto para mejorar la extracción del producto y el diseño geométrico de la briqueta. 
En base a los cálculos efectuados se encontró que el nivel de producción de la máquina hidráulica anualmente es de 141,953 unidades, en cambio la prensa manual de hierro produce 37,597 unidades anuales, encontrando una diferencia de 104,356 unidades, esto indica que la productividad de la máquina hidráulica es mayor que de la prensa manual de hierro, de esta manera se respondió a la hipótesis planteada "El funcionamiento de la máquina hidráulica es más eficiente y productivo, que el de la prensa manual de hierro"; por otro lado la máquina hidráulica mejora otros aspectos como el diseño geométrico de las briquetas y favorece el manejo del operario. Posteriormente se procedió a elaborar un manual de uso y mantenimiento que facilite información necesaria para operar el equipo; en dicho manual se abordaron aspectos como el fabricante, seguridad, descripción del equipo, instrucciones de utilización y los diferentes mantenimientos que se le debe dar al equipo.

Con respecto a la caracterización de las briquetas se obtuvo que de las 18 mezclas planteadas resulto 4 mezclas óptimas, las cuales son la EXM1, EXPM2, EXPM4 y EXPM5 (Ver figuras 1, 2, 3 y 4); a dichas mezclas se le realizaron pruebas físicas y se compararon con la mezcla testigo y la leña; teniendo como resultado lo siguiente:

- EXPM1: \% de ceniza: 1.9\%, \% promedio de humedad: $2.77 \%$, CO2: 535.75 ppm, tiempo de ebullición: 13 min y tiempo de combustión: 32.31 min.

- EXPM2: \% de ceniza: 3.28\%, \% promedio de humedad: $3.09 \%$, CO2: 526 ppm, tiempo de ebullición: 16.25 min y tiempo de combustión: 25.35 min.

- EXPM4: \% de ceniza: 2.81\%, \% promedio de humedad: $3.77 \%$, CO2: 610 ppm, tiempo de ebullición: 19.5 min y tiempo de combustión: 22.89 min.

- EXPM5: \% de ceniza: 2.89\%, \% promedio de humedad: $2.61 \%$, CO2: 607.75 ppm, tiempo de ebullición: 17.75 min y tiempo de combustión: 21.58 min.

- Mezcla testigo: \% de ceniza: 1\%, \% promedio de humedad: $7 \%$, CO2: $700 \mathrm{ppm}$, tiempo de ebullición: 20 min y tiempo de combustión: 15 min.

- Leña: \% de ceniza: 1\%, CO2: 955.5 ppm, tiempo de ebullición: 32.25 min y tiempo de combustión: 48.25 min.

La comparación entre los tratamientos efectivos, la mezcla testigo y la leña donde se observa que: la mezcla testigo es la que produce menor porcentaje de cenizas, la EXPM5 posee el menor porcentaje de humedad, la EXPM2 genera menor cantidad de $\mathrm{CO}_{2}$, la EXPM1 tiene el menor tiempo de ebullición y la leña posee el mayor tiempo de combustión; pero la EXPM1 posee una diferencia mínima en comparación con los datos de la mezcla testigo, la leña, la EXPM5 y EXPM2 lo que significa que la mejor de las opciones para consumo como combustible es la EXPM1, seguido por la EXPM2, como se muestra en la figura 3 y 4. 


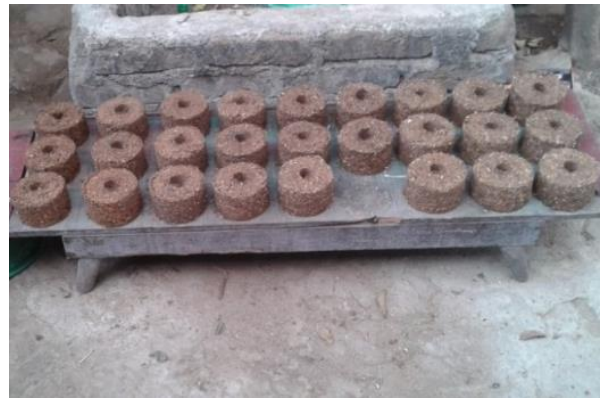

Fig. 3: Briquetas de la EXPM1

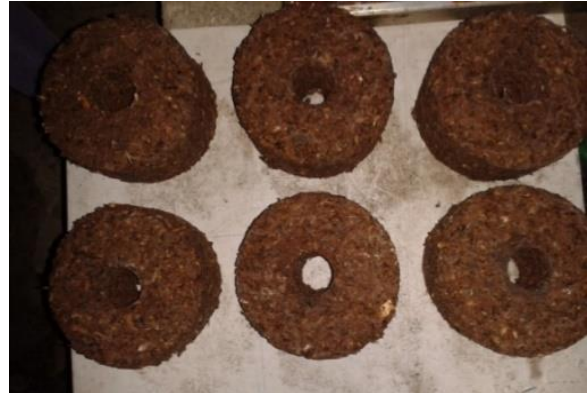

Fig. 4: Briquetas de la EXPM2

En base a los tratamientos EXPM1 y EXPM2 se calculó la productividad de la máquina, teniendo un tiempo promedio de 6.96 min por briqueta para EXPM1 elaborando 416 briquetas al día, 10,816 briquetas mensual y 129,792 anual. Para la EXPM2 el tiempo promedio es de 7.43 min por briqueta, produciendo 384 briquetas al día, 2,304 briquetas al mes y 9,984 briquetas anual.

Tomando de referencia los datos obtenidos del cálculo de productividad de la máquina hidráulica utilizando la mezcla óptima "Cisco sin moler + aserrín + almidón + agua" (EXPM1) y el $85 \%$ de efectividad de mano de obra; al día se producirían 353 briquetas, mensualmente 9,193 briquetas y anualmente 110,323. De acuerdo a estos datos para la producción de 110,323 briquetas anuales se generarían un costo total de producción C $\$ 497,731.49$ por ende se obtiene un costo de producción de $C \$ 4.51$ por unidad de producción (6 briquetas), que equivale a un costo unitario de $\mathrm{C} \$ 0.75$ por briqueta.

Los costos de producción utilizando la mezcla óptima número dos "Cisco sin moler + aserrín + colocho + almidón + agua” (EXPM2) y tomando el 85 \% de efectividad de mano de obra, al día se producirían 326 briquetas, mensualmente 8,486 briquetas y anualmente 101,836; de acuerdo a esto para la producción de 101,836 briquetas anuales se generarían un costo total de producción $C \$ 471,287.47$ por ende se obtiene un costo de producción de $C \$ 4.62$ por unidad de producción ( 6 briquetas), que equivale a un costo unitario de $\mathrm{C} \$ 0.77$ por briqueta.

\section{CONCLUSIONES}

La máquina hidráulica es más productiva en comparación a la prensa manual de hierro, ya que produce mayor cantidad de briquetas en un tiempo de trabajo (6.33 min produce 1 unidad) menor; siendo 141,953 de briquetas anuales para la máquina hidráulica y 37,597 unidades de briquetas para la prensa manual de hierro. La máquina hidráulica supera a la prensa manual de hierro en aproximadamente 4 veces la cantidad de briquetas producidas al año, además con la misma se logró mejorar la geometría de la briqueta, que en consecuencia favorece la operación de secado y la combustión de las mismas. Así mismo cabe destacar que la máquina hidráulica mejora la ergonomía postural del operador, es de fácil manipulación, alimentación, el espacio de trabajo que ocupa es poco y la limpieza del equipo no es complicada. 
Por otra parte las briquetas elaboradas con materias primas molido y sin moler más residuos de madera (Aserrín y Colocho) fueron sometidas a diferentes pruebas físicas (Durabilidad de Combustión, Porcentaje de Humedad, Porcentaje de Ceniza, Poder Calorífico y Emisión de $\mathrm{CO}_{2}$ ), resultando que los tratamientos: EXPM1 Y EXPM2 son mejores que la leña y la mezcla testigo ya que producen menos dióxido de carbono, su tiempo de durabilidad es mayor, su tiempo de encendido es menor pero su poder calorífico es mayor debido al diseño geométrico de la briqueta.

En los costos de producción se tuvo como resultado que las briquetas de la EXPM1 cuestan C\$ 4.51 por unidad ( 6 briquetas) y las de la EXPM2 cuestan $C \$ 4.62$ (6 briquetas), en cambio 1 briqueta producida en la prensa manual de hierro cuesta $C \$ 4.50$.

\section{REFERENCIAS}

Amador, A., Rugama, J., \& Morazán, F. (2012). Validación de un prototipo de una máquina briqueteadora industrial para el aprovechamiento de la cascarilla de café en CISA, Exportadora, Sébaco-Matagalpa. Estelí, Nicaragua: UNI.

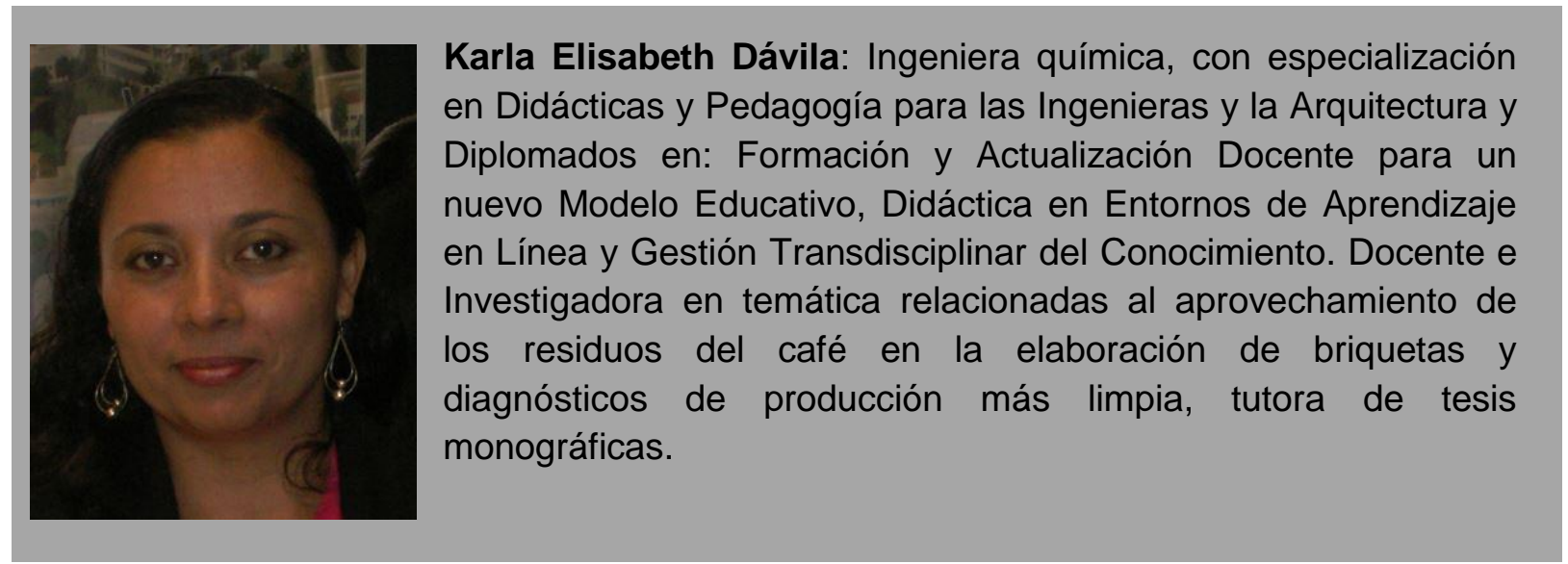

